Physics

Physics Research Publications

\title{
Quantized quasi-two-dimensional Bose-Einstein condensates with spatially modulated nonlinearity
}
D. S. Wang
X. H. Hu
J. P. Hu
W. M. Liu

This paper is posted at Purdue e-Pubs.

http://docs.lib.purdue.edu/physics_articles/1336 


\title{
Quantized quasi-two-dimensional Bose-Einstein condensates with spatially modulated nonlinearity
}

\author{
Deng-Shan Wang, ${ }^{1}$ Xing-Hua Hu, ${ }^{1}$ Jiangping Hu, ${ }^{2}$ and W. M. Liu ${ }^{1}$ \\ ${ }^{1}$ Beijing National Laboratory for Condensed Matter Physics, Institute of Physics, Chinese Academy of Sciences, \\ Beijing 100190, People's Republic of China \\ ${ }^{2}$ Department of Physics, Purdue University, West Lafayette, Indiana 47907, USA
}

(Received 3 December 2009; published 25 February 2010)

\begin{abstract}
We investigate the localized nonlinear matter waves of the quasi-two-dimensional Bose-Einstein condensates with spatially modulated nonlinearity in the harmonic potential. It is shown that all of the Bose-Einstein condensates, similar to the linear harmonic oscillator, can have an arbitrary number of localized nonlinear matter waves with discrete energies, which are mathematically exact orthogonal solutions of the Gross-Pitaevskii equation. Their properties are determined by the principal quantum number $n$ and secondary quantum number $l$ : the parity of the matter wave functions and the corresponding energy levels depend only on $n$, and the numbers of density packets for each quantum state depend on both $n$ and $l$, which describe the topological properties of the atom packets. We also give an experimental protocol to observe these phenomena in future experiments.
\end{abstract}

DOI: 10.1103/PhysRevA.81.025604

PACS number(s): 03.75.Hh, 05.45.Yv, 67.85.Bc

Introduction. Since the remarkable experimental realization [1-3] of Bose-Einstein condensates (BECs), there has been an explosion of experimental and theoretical activity devoted to the physics of dilute ultracold bosonic gases. It is known that the properties of BECs including their shape and collective nonlinear excitations are determined by the sign and magnitude of the $s$-wave scattering length. A prominent way to adjust scattering length is to tune an external magnetic field in the vicinity of a Feshbach resonance [4]. Alternatively, one can use a Feshbach resonance induced by an optical or electric field [5]. Since all quantities of interest in the BEC crucially depend on scattering length, a tunable interaction suggests very interesting studies of the many-body behavior of condensate systems.

In past years, techniques for adjusting the scattering length globally have been crucial to many experimental achievements $[6,7]$. More recently, condensates with a spatially modulated nonlinearity by manipulating scattering length locally have been proposed [8-12]. This is experimentally feasible due to the flexible and precise control of the scattering length with tunable interactions. The spatial dependence of scattering length can be implemented by a spatially inhomogeneous external magnetic field in the vicinity of a Feshbach resonance [13].

However, so far, the studies of BECs with spatially modulated nonlinearity are limited to the quasi-one-dimensional cases [8-12]. Moreover, in the study of nonlinear problems no one discusses their quantum properties, which are common in linear systems such as the linear harmonic oscillator. In this Brief Report, we extend the similarity transformation [12] to the quasi-two-dimensional (quasi-2D) BECs with spatially modulated nonlinearity in harmonic potential, and find a family of localized nonlinear matter wave solutions. Similar to the linear harmonic oscillator, we discover that the whole BEC can be quantized, which was unexpected before. Their quantum and topological properties can be simply described by two quantum numbers. We also formulate an experimental procedure for the realization of these phenomena in ${ }^{7} \mathrm{Li}$ condensate [2,14]. This opens the door to the investigation of new matter waves in the high-dimensional BEC with spatially modulated nonlinearities.
Model and exact localized solutions. The system considered here is a BEC confined in a harmonic trap $V(\mathbf{r})=$ $m\left(\omega_{\perp}^{2} r^{2}+\omega_{z}^{2} z^{2}\right) / 2$, where $m$ is atomic mass, $r^{2}=x^{2}+y^{2}$, and $\omega_{\perp}, \omega_{z}$ are the confinement frequencies in the radial and axial directions, respectively. In the mean-field theory, the BEC system at low temperature is described by the Gross-Pitaevskii (GP) equation in three dimensions. If the trap is pancake shaped, that is, $\omega_{z} \gg \omega_{\perp}$, it is reasonable to reduce the GP equation for the condensate wave function to a quasi-2D equation [15-17]:

$$
i \psi_{t}=-\frac{1}{2}\left(\psi_{x x}+\psi_{y y}\right)+\frac{1}{2} \omega^{2}\left(x^{2}+y^{2}\right) \psi+g(x, y)|\psi|^{2} \psi,
$$

where $\omega=\omega_{\perp} / \omega_{z}$, the length, time, and wave function $\psi$ are measured in units of $a_{h}=\sqrt{\hbar / m \omega_{z}}, \omega_{z}^{-1}, a_{h}^{-1}$, and $g(x, y)=$ $4 \pi a_{s}(x, y)$ represents the strength of interatomic interaction characterized by the $s$-wave scattering length $a_{s}(x, y)$, which can be spatially inhomogeneous by magnetically tuning the Feshbach resonances [4,8-13].

Now we consider the spatially localized stationary solution $\psi(x, y, t)=\phi(x, y) e^{-i \mu t}$ of Eq. (1) with $\phi(x, y)$ being a real function for $\lim _{|x|,|y| \rightarrow \infty} \phi(x, y)=0$. This maps Eq. (1) onto a stationary nonlinear Schrödinger equation $\frac{1}{2} \phi_{x x}+\frac{1}{2} \phi_{y y}-$ $\frac{1}{2} \omega^{2}\left(x^{2}+y^{2}\right) \phi-g(x, y) \phi^{3}+\mu \phi=0$ [18]. Here $\mu$ is the real chemical potential. Solving this stationary equation by similarity transformation [12], we obtain a family of exact localized nonlinear wave solutions for Eq. (1) as

$$
\begin{array}{ll}
\psi_{n}=\frac{(n+1) K(k) \eta}{\sqrt{v}} \operatorname{cn}(\theta, k) e^{-i \mu t}, & n=0,2,4, \ldots \\
\psi_{n}=\frac{(n+1) K(k) \eta}{\sqrt{2 v}} \operatorname{sd}(\theta, k) e^{-i \mu t}, & n=1,3,5, \ldots
\end{array}
$$

where $k=\sqrt{2} / 2$ is the modulus of elliptic function, $v$ is a positive real constant, $K(k)=\int_{0}^{\frac{\pi}{2}}\left[1-k^{2} \sin ^{2} \varsigma\right]^{-1 / 2} d \varsigma$ is elliptic integral of the first kind, sd $=\mathrm{sn} / \mathrm{dn}$ with sn, $\mathrm{cn}$, and dn being Jacobi elliptic functions, $\theta, \eta$, and $g$ are determined by

$$
\begin{gathered}
\theta=(n+1) K(k) \operatorname{erf}[\sqrt{2 \omega}(x+y) / 2], \\
\eta=e^{\omega x y} U\left[-\mu /(2 \omega), 1 / 2, \omega(x-y)^{2} / 2\right], \\
g(x, y)=-2 \omega \nu /\left(\pi \eta^{2}\right) e^{-\omega(x+y)^{2}},
\end{gathered}
$$


where $\operatorname{erf}(x)=\frac{2}{\sqrt{\pi}} \int_{0}^{x} e^{-\tau^{2}} d \tau$ is the error function, and $U(a, c, s)$ [19] is the Kummer $U$ function of the second kind, which is a solution of ordinary differential equation $s \Lambda^{\prime \prime}(s)+(c-s) \Lambda^{\prime}(s)-a \Lambda(s)=0$. It is easy to see that when $|x|,|y| \rightarrow \infty$ we have $\psi_{n} \rightarrow 0$ for solutions $\psi_{n}$ in Eqs. (2) and (3) with Eq. (4), thus they are localized bound state solutions.

In the above construction, it is observed that the number of zero points of function $\eta$ in Eq. (4) is equal to that of function Kummer $U\left[-\mu /(2 \omega), 1 / 2, \omega(x-y)^{2} / 2\right]$, which strongly depends on $\omega$ and the ratio $\mu / \omega$. We assume the number of zero points in $\eta$ along line $y=-x$ is $l$. In the following, we will see that integer $n$ is associated with the energy levels of the atoms and integers $n, l$ determine the topological properties of atom packets, so $n$ and $l$ are named the principal quantum number and secondary quantum number in quantum mechanics. In addition, the three free parameters $\omega$, $\mu$, and $\nu$ are positive, so the dimensionless interaction function $g(x, y)$ is negative, which indicates an attractive interaction between atoms. There are known atomic gases with attractive interactions realized by modulating magnetic [4] technique, for example, the ${ }^{85} \mathrm{Rb}[20]$ and ${ }^{7} \mathrm{Li}$ atoms $[2,14]$.

To translate our results into units relevant to the experiments $[2,14]$, we take the ${ }^{7} \mathrm{Li}$ condensate containing $10^{3}-10^{5}$ atoms in a pancake-shaped trap with radial frequency $\omega_{\perp}=2 \pi \times$ $10 \mathrm{~Hz}$ and axial frequency $\omega_{z}=2 \pi \times 500 \mathrm{~Hz}$ [21]. In this case, the ratio of trap frequency $\omega$ in Eq. (1) is 0.02 , which is determined by $\omega_{\perp} / \omega_{z}$. The unit of length is $1.69 \mu \mathrm{m}$, the unit of time is $0.32 \mathrm{~ms}$, and the unit of chemical potential is $\mathrm{nK}$. The spatially inhomogeneous interaction parameter $g(x, y)$ is independent of principal quantum number $n$ but is strongly related to the secondary quantum number $l$. In Fig. 1, we show that for $\omega=0.02, v=0.1$, function $g(x, y)$ is smooth in space when $l=0$ and develops singularity when the $l$ gets large.

Quantized quasi-2D BEC. In order to investigate the quantum and topological properties of the localized nonlinear matter waves in quasi-2D BECs described by Eqs. (2) and (3) with Eq. (4), we plot their density distributions by

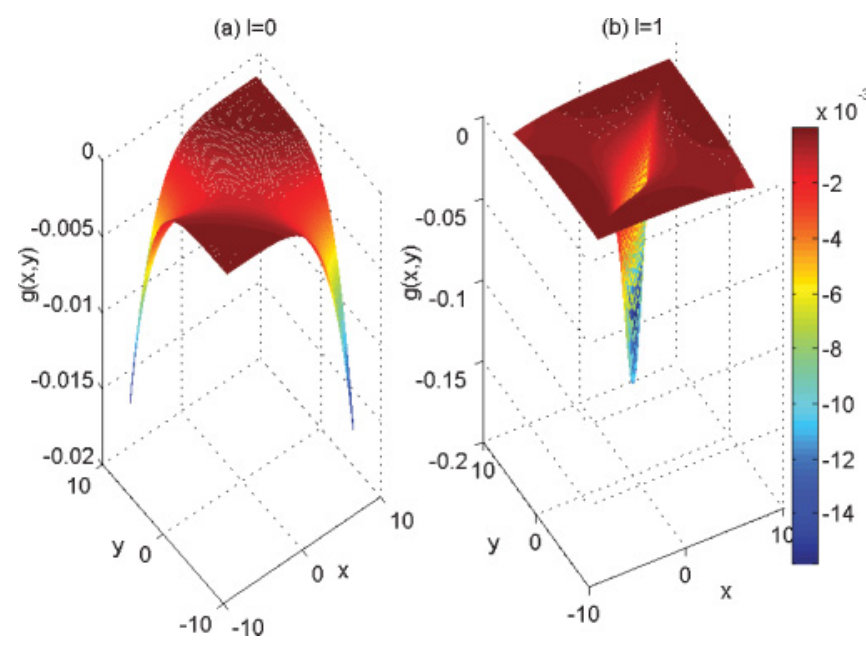

FIG. 1. (Color online) The interaction parameter $g(x, y)$ for two secondary quantum numbers: (a) $l=0$ and (b) $l=1$ with $\omega=0.02, \quad v=0.1$. It is seen that $g(x, y)$ is a smooth function when $l=0$ and develops singularity when $l$ gets large. Here, the unit of space length $x, y$ is $1.69 \mu \mathrm{m}$.
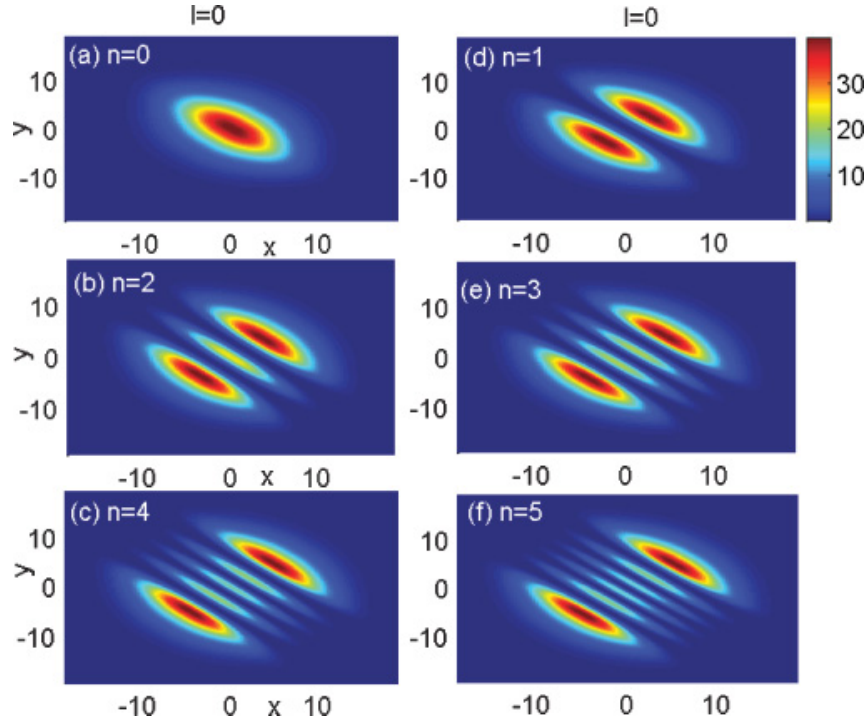

FIG. 2. (Color online) The density distributions of the quasi-2D BEC with spatially modulated nonlinearities in harmonic potential, for different principle quantum numbers $n$ in Eqs. (2) and (3) with Eq. (4), where the secondary quantum number $l=0$ and the parameters $\omega, v$ are 0.02 and 0.1 , respectively. The unit of space length $x, y$ is $1.69 \mu \mathrm{m}$. Figs. 2(a)-2(c) show the density profiles of the even parity wave function (2) for $n=0,2$, and 4 , respectively. Figs. 2(d)-2(f) demonstrate the density profiles of the odd parity wave function (3) for $n=1,3$ and 5, respectively.

manipulating the principal quantum number $n$ or secondary quantum number $l$.

Firstly, when the secondary quantum number $l$ is fixed, we can modulate the principal quantum number $n$ to analyze the novel matter waves in quasi-2D BEC. Figure 2 shows the density profiles in quasi-2D BEC with spatially modulated nonlinearities in harmonic potential for $l=0$. It is easy to see that the matter wave functions in Eq. (2) satisfy $\psi_{n}(-x,-y)=$ $\psi_{n}(x, y)$, so they are even parity and are invariant under space inversion. Figures 2(a)-2(c) demonstrate the density profiles of the even parity wave functions (2) with Eq. (4) for $n=0,2,4$, which correspond to a low energy state and two highly excited states. The numbers of atoms $N_{n}=\iint d x d y\left|\psi_{n}(x, y, t)\right|^{2}$ for the three states are $N_{0}=3.76 \times 10^{3}, N_{2}=6.84 \times$ $10^{4}, N_{4}=2.633 \times 10^{5}$, respectively. The matter wave functions in Eq. (3) satisfy $\psi_{n}(-x,-y)=-\psi_{n}(x, y)$, which denotes that they are odd parity. Figs. 2(d)-2(f) demonstrate the density profiles of the odd parity wave functions (3) with Eq. (4) for $n=1,3,5$, which correspond to three highly excited states. The numbers of atoms for the three states are $N_{1}=4.016 \times 10^{4}, N_{3}=2.493 \times 10^{5}, N_{5}=7.28 \times 10^{5}$, respectively. It is observed that when the secondary quantum number $l=0$, the number of nodes along line $y=x$ for each quantum state is equal to the corresponding principal quantum number $n$ (i.e., the $n$th level quantum state has $n$ nodes along $y=x)$. And the number of density packets increases one by one along line $y=x$ when the $n$ increases. This is similar to the quantum properties in the linear harmonic oscillator.

Secondly, when the principal quantum number $n$ is fixed, we can tune the secondary quantum number $l$ to observe 

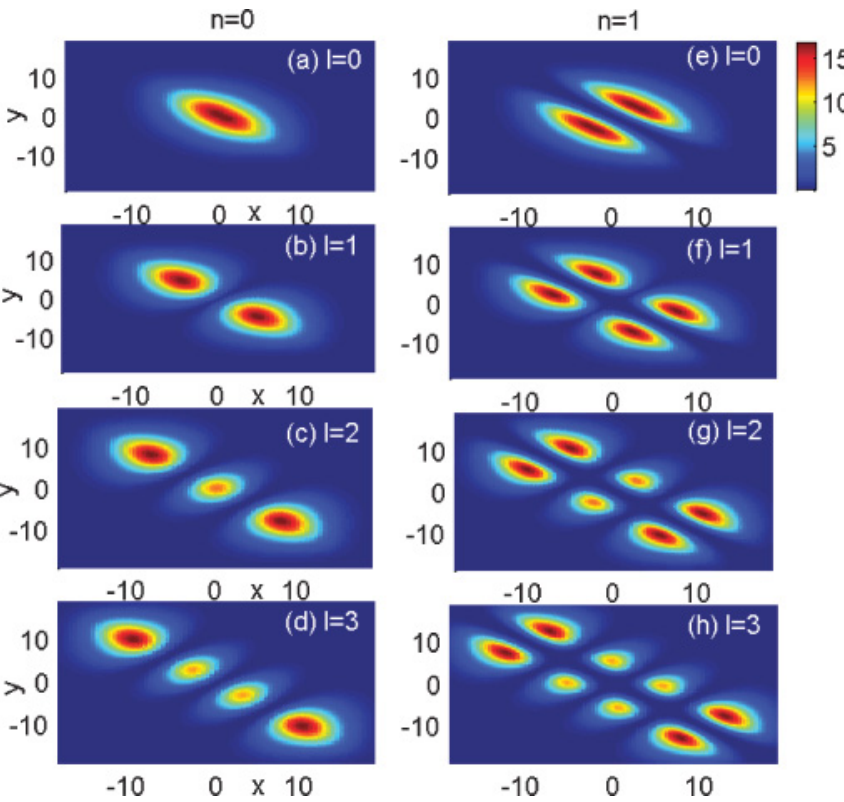

FIG. 3. (Color online) The density distributions of the quasi-2D $\mathrm{BEC}$ in harmonic potential for different secondary quantum number l. Figs. 3(a)-3(d) show the density profiles of the even parity wave function (2) for principal quantum number $n=0$, and Figs. 3(e)-3(h) show the density profiles of the odd parity wave function (3) for $n=1$, corresponding to $l=0,1,2,3$. The other parameters are the same as that of Fig. 2. The unit of space length is $1.69 \mu \mathrm{m}$.

the novel quantum phenomenon in quasi-2D BEC. In Fig. 3 we demonstrate the density distributions of quasi-2D BEC in harmonic potential for different secondary quantum number. Figures 3(a)-3(d) show the density profiles of the even parity wave function (2) with Eq. (4) for $n=0$, and $l=0,1,2$, and 3 , respectively. It is seen that the number of nodes for the density packets along line $y=-x$ is equal to the corresponding secondary quantum number $l$, which describes the topological patterns of the atom packets, and the number of density packets increases one by one when $l$ increases. Figures $3(\mathrm{e})-3(\mathrm{~h})$ show the density profiles of the odd parity wave function (3) with Eq. (4) for $n=1$ and $l=0,1,2,3$. We see that the number of density packets increases pair by pair when $l$ increases. The number of density packets for each quantum state is equal to $(n+1) \times(l+1)$, and all the density packets are symmetrical with respect to lines $y= \pm x$, as shown in Figs. 2 and 3.

Normalization energy versus chemical potential. Next we calculate the normalization energy of each quantum state numerically. The total energy of the quasi-2D BEC is $E(\psi)=$ $\iint d x d y\left[|\nabla \psi|^{2}+\frac{1}{2} \omega^{2}\left(x^{2}+y^{2}\right)|\psi|^{2}+\frac{1}{2} g(x, y)|\psi|^{4}\right]$. Therefore, the normalized energy is given by $E(\psi) / N=\mu-$ $\frac{1}{2 N} \iint d x d y g(x, y)|\psi|^{4}$ with $N=\iint d x d y|\psi|^{2}$. Figure 4 shows the relations of the normalization energy $E(\psi) / N$ with chemistry potential for different principle quantum numbers $n$. It is observed that for the fixed $n$, the normalization energy is an approximatively linear increase with respect to chemistry potential, that is, $d(E(\psi) / N) / d \mu>0$. Figure 4(a) demonstrates that the normalization energy for the even parity wave function (2) increases when the principal quantum number $n$ increases. So does the odd parity wave (a)
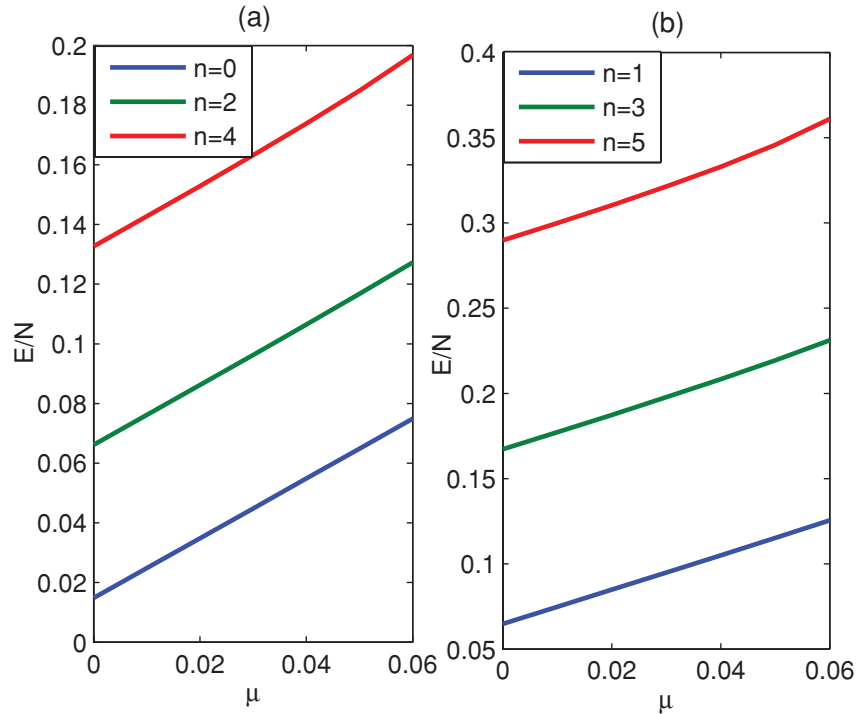

FIG. 4. (Color online) The normalization energy $E(\psi) / N$ versus chemical potential $\mu$, with $N=\iint d x d y|\psi|^{2}$. (a) Even parity wave function (2) with principal quantum numbers $n=0,2,4$ and (b) odd parity wave function (3) with $n=1,3,5$. The parameters are $\omega=0.02$ and $v=0.1$. Here, the curves in the legend are shown from bottom to top in the figures.

function (3), as shown in Fig. 4(b). It is shown that the energy levels of the atoms are only associated with the principal quantum number $n$. These are similar to energy level distribution of the energy eigenvalue problem for the linear harmonic oscillator described by linear Schrödinger equation.

Stability analysis. Stability of exact solutions with respect to perturbation is very important, because only stable localized nonlinear matter waves are promising for experimental observations and physical applications. To study the stability of our exact solutions (2) and (3) with Eq. (4), we consider a perturbed solution $\psi(x, y, t)=\left[\phi_{n}(x, y)+\Psi(x, y, t)\right] e^{-i \mu t}$ of Eq. (1). Here, $\phi_{n}(x, y)$ are the exact solutions of the stationary nonlinear Schrödinger equation $\frac{1}{2} \phi_{x x}+\frac{1}{2} \phi_{y y}-\frac{1}{2} \omega^{2}\left(x^{2}+y^{2}\right) \phi-$ $g(x, y) \phi^{3}+\mu \phi=0 . \Psi(x, y, t) \ll 1$ is a small perturbation to the exact solutions and $\Psi(x, y, t)=[R(x, y)+I(x, y)] e^{i \lambda t}$ is decomposed into its real and imaginary parts [23]. Substituting this perturbed solution to the quasi-2D GP equation (1) and neglecting the higher-order terms in $(R, I)$, we obtain a standard eigenvalue problem $L_{+} R=\lambda I, L_{-} I=\lambda R$, where $\lambda$ is eigenvalue, $R, I$ are eigenfunctions with $L_{+}=-\frac{1}{2}\left(\partial_{x}^{2}+\partial_{y}^{2}\right)+$ $3 g(x, y) \phi_{n}(x, y)^{2}+\frac{1}{2} \omega^{2}\left(x^{2}+y^{2}\right)-\mu$, and $L_{-}=-\frac{1}{2}\left(\partial_{x}^{2}+\right.$ $\left.\partial_{y}^{2}\right)+g(x, y) \phi_{n}(x, y)^{2}+\frac{1}{2} \omega^{2}\left(x^{2}+y^{2}\right)-\mu$. Numerical experiments show that when $\omega=0.02$ and $\mu, v$ are arbitrary non-negative constants, only for principle quantum number $n=0$ is the eigenvalue $\lambda$ of this eigenvalue problem real. This suggests that for $\omega=0.02$ the exact localized nonlinear matter wave solution (2) is linearly stable only for $n=0$ and solution (3) is linearly unstable for all $n$ (see Fig. 5). It is seen that when the frequencies of the pancake-shaped trap is fixed, the stability of the exact solutions (2) and (3) with Eq. (4) rests only on the principal quantum number $n$.

Experimental protocol. We now provide an experimental protocol for creating the quasi-2D localized nonlinear matter waves. To do so, we take the attractive ${ }^{7} \mathrm{Li}$ condensate 


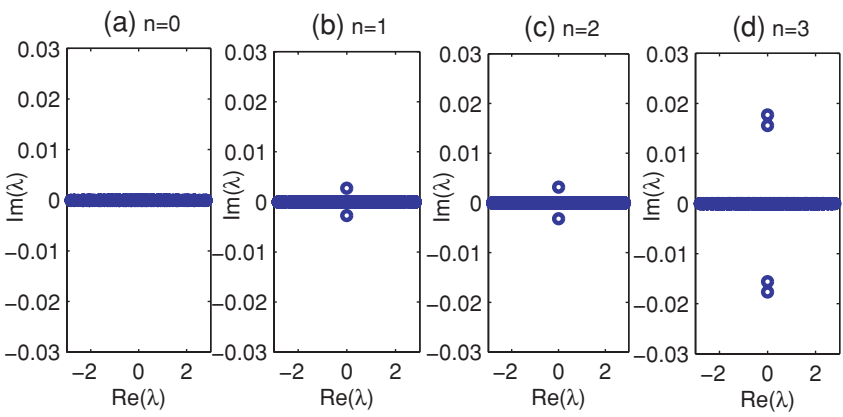

FIG. 5. (Color online) Eigenvalue for different principal quantum numbers $n$ with parameters $\omega=0.02, \mu=0.001$, and $v=0.1$. It is shown that only for $n=0$ is the localized nonlinear matter wave solution (2) linearly stable, and the solution (3) is linearly unstable for all $n$.

[2,14], containing about $10^{3}-10^{5}$ atoms, confined in a pancake-shaped trap with radial frequency $\omega_{\perp}=2 \pi \times 10 \mathrm{~Hz}$ and axial frequency $\omega_{z}=2 \pi \times 500 \mathrm{~Hz}$ [21]. This trap can be determined by a combination of spectroscopic observations, direct magnetic field measurement, and the observed spatial cylindrical symmetry of the trapped atom cloud [21]. The next step is to realize the spatial variation of the scattering length. Near the Feshbach resonance [4,13,16,24], the scattering length $a_{s}(B)$ varies dispersively as a function of magnetic field $B$, that is, $a_{s}(B)=a\left[1+\Delta /\left(B_{0}-B\right)\right]$, with $a$ being the asymptotic value of the scattering length far from the resonance, $B_{0}$ being the resonant value of the magnetic field, and $\Delta$ being the width of the resonance. For the magnetic field in $z$ direction with gradient $\alpha$ along $x-y$ direction, we have $\vec{B}=\left[B_{0}+\alpha B_{1}(x, y)\right] \vec{e}_{z}$. In this case, the scattering length is dependent on $x$ and $y$. In real experiments, the spatially dependent magnetic field may be generated by a microfabricated ferromagnetic structure integrated on an atom chip [25,26], such that interaction in Fig. 1 can be realized. In order to observe the density distributions in Figs. 2 and 3 clearly in experiment, the ${ }^{7} \mathrm{Li}$ atoms should be evaporatively cooled to low temperatures, say in the range of $10-100 \mathrm{nK}$. After the interaction parameter in Fig. 1(a) is realized by modulating magnetic field properly, the density distributions in Fig. 2 can be observed for different numbers of atoms by evaporative cooling, for example, the numbers of atoms in Fig. 2(a)-2(c) are $3.76 \times 10^{3}, 6.84 \times 10^{4}$, and $2.633 \times 10^{5}$, respectively. The density distributions in Fig. 3 can also be observed by changing the scattering lengths through magnetic field for various atom numbers.

Conclusion. In summary, we have discovered a family of exact localized nonlinear matter wave solutions of the quasi-2D BEC with spatially modulated nonlinearities in harmonic potential. Similar to the linear harmonic oscillator, we introduce two classes of quantum numbers: the principal quantum number $n$ and secondary quantum number $l$. The matter wave functions have even parity for the even principal quantum number and odd parity for the odd one, the energy levels of the atoms are only associated with the principal quantum number, and the number of density packets for each quantum state is equal to $(n+1) \times(l+1)$. We also provide an experimental scheme to observe these phenomena in future experiments. Our results are of particular significance to matter wave management in high-dimensional BEC.

The authors thank H. Pu, Q. Niu, G. C. Liu, Y. P. Zhang, and $\mathrm{B}$. $\mathrm{Xu}$ for useful discussions. This work was supported by NSFC under Grants No. 10874235, No. 10934010, and No. 60978019, and by NKBRSFC under Grants No. 2006CB921400, No. 2009CB930704, and No. 2010CB922904. D. S. Wang was supported by the China Postdoctoral Science Foundation.
[1] M. H. Anderson et al., Science 269, 198 (1995).

[2] C. C. Bradley et al., Phys. Rev. Lett. 75, 1687 (1995).

[3] K. B. Davis et al., Phys. Rev. Lett. 75, 3969 (1995).

[4] S. Inouye et al., Nature 392, 151 (1998); J. L. Roberts et al., Phys. Rev. Lett. 81, 5109 (1998); Z. X. Liang, Z. D. Zhang, and W. M. Liu, ibid. 94, 050402 (2005).

[5] M. Marinescu and L. You, Phys. Rev. Lett. 81, 4596 (1998); M. Theis et al., ibid. 93, 123001 (2004).

[6] L. Khaykovich et al., Science 296, 1290 (2002); J. Herbig et al., ibid. 301, 1510 (2003).

[7] M. Bartenstein et al., Phys. Rev. Lett. 92, 203201 (2004).

[8] M. I. Rodas-Verde, H. Michinel, and V. M. Perez-Garcia, Phys. Rev. Lett. 95, 153903 (2005); A. V. Carpentier et al., Phys. Rev. A 74, 013619 (2006).

[9] H. Sakaguchi and B. A. Malomed, Phys. Rev. E 72, 046610 (2005); G. Theocharis et al., Phys. Rev. A 72, 033614 (2005); F. K. Abdullaev and J. Garnier, ibid. 72, 061605(R) (2005); J. Garnier and F. K. Abdullaev, ibid. 74, 013604 (2006); M. A. Porter et al., Physica D 229, 104 (2007); D. A. Zezyulin et al., Phys. Rev. A 76, 013621 (2007).

[10] M. Salerno, V. V. Konotop, and Y. V. Bludov, Phys. Rev. Lett. 101, 030405 (2008); V. A. Brazhnyi et al., ibid. 102, 144101 (2009).
[11] G. Dong and B. Hu, Phys. Rev. A 75, 013625 (2007); L. C. Qian et al., ibid. 77, 013611 (2008).

[12] J. Belmonte-Beitia et al., Phys. Rev. Lett. 98, 064102 (2007); 100, 164102 (2008); A. T. Avelar, D. Bazeia, and W. B. Cardoso, Phys. Rev. E 79, 025602(R) (2009).

[13] H. Xiong et al., Phys. Rev. Lett. 95, 120401 (2005).

[14] J. M. Gerton et al., Nature London 408, 692 (2000); M. Junker et al., Phys. Rev. Lett. 101, 060406 (2008); S. E. Pollack et al., ibid. 102, 090402 (2009).

[15] G. Theocharis et al., Phys. Rev. Lett. 90, 120403 (2003); X. H. Hu et al., Phys. Rev. A 79, 023619 (2009).

[16] H. Saito and M. Ueda, Phys. Rev. Lett. 90, 040403 (2003).

[17] J. J. Garcia-Ripoll et al., Phys. Rev. Lett. 87, 140403 (2001)

[18] G. Baym and C. J. Pethick, Phys. Rev. Lett. 76, 6 (1996).

[19] M. Abramowitz and I. Stegun, Handbook of Mathematical Functions (Dover, New York, 1965).

[20] S. L. Cornish et al., Phys. Rev. Lett. 85, 1795 (2000).

[21] D. Rychtarik et al., Phys. Rev. Lett. 92, 173003 (2004).

[22] P. A. Ruprecht et al., Phys. Rev. A 51, 4704 (1995).

[23] J. C. Bronski et al., Phys. Rev. Lett. 86, 1402 (2001).

[24] F. K. Abdullaev et al., Phys. Rev. Lett. 90, 230402 (2003).

[25] M. Vengalattore et al., J. Appl. Phys. 95, 4404 (2004).

[26] M. Vengalattore et al., Eur. Phys. J. D 35, 69 (2005). 